\title{
HELMINTOS DO CACHORRO DO CAMPO, Pseudalopex gymnocercus (FISCHER, 1814) E DO CACHORRO DO MATO, Cerdocyon thous (LINNAEUS, 1766) NO SUL DO ESTADO DO RIO GRANDE DO SUL, BRASIL
}

\author{
JERÔNIMO L. RUAS ${ }^{1}$; GERTRUD MULLER²; NARA AMÉLIA R. FARIAS ${ }^{2}$; TIAGO GALLINA ${ }^{3}$; ANDREIA S. LUCAS ${ }^{3}$; \\ FELIPE G. PAPPEN ${ }^{3}$; AFONSO L. SINKOC ${ }^{4}$; JOÃO GUILHERME W. BRUM ${ }^{2}$
}

\begin{abstract}
RUAS, J.L.; MULLER, G.; FARIAS, N.A.R.; GALLINA, T.; LUCAS, A.S.; PAPPEN, F.G.; SINKOC, A.L.; BRUM, J. G.W. [Helminths of Pampas fox Pseudalopex gymnocercus (Fischer, 1814) and of Crab-eating fox Cerdocyon thous (Linnaeus, 1766) in the Southern of the State of Rio Grande do Sul, Brazil]. Helmintos do Cachorro do campo Pseudalopex gymnocercus (Fischer, 1814) e do Cachorro do mato Cerdocyon thous (Linnaeus, 1766) no sul do estado do Rio Grande do Sul, Brasil. Revista Brasileira de Parasitologia Veterinária, v. 17, n. 2, p. 87-92, 2008. Laboratório Regional de Diagnóstico, Faculdade de Veterinária, Universidade Federal de Pelotas, Rio Grande do Sul, Brasil. Caixa Posta 354, CEP.: 96.010-900. E-mail: ruas@ ufpel.edu.br

Forty wild canids were captured by live trap at Municipalities of Pedro Osorio and Pelotas in Southern of the State of Rio Grande do Sul and they were transported to the Parasitology Laboratory at the Universidade Federal de Pelotas. After they were posted, segments of intestinal, respiratory and urinary tracts and liver were separated and examined. Animal skulls were used for taxonomic identification. Of forty wild animals trapped, 22 (55\%) were Pseudalopex gymnocercus and 22 (55\%) Cerdocyon thous. The most prevalent nematodes were: Ancylostoma caninum (45.4 in P. gymnocercus and 22.2\% in C. thous), Molineus felineus (9.9 in P. gymnocercus and 5.6\% in C. thous), Strongyloides sp. (22.7 in P. gymnocercus and 16.7\% in C. thous), Trichuris sp. (13.6 in P. gymnocercus and $11.1 \%$ in C. thous), and Capillaria hepatica (13.6 in P. gymnocercus and $5.5 \%$ in C. thous). The trematodes observed were: Alaria alata (36.4 in P. gymnocercus and 50.0\% in C. thous), and Asthemia heterolecithodes in $5.6 \%$ C. thous. Cestodes were identified as Spirometra sp. (61.1\% in C. thous and 54.5 in P. gymnocercus), Diphyllobothriidae, (81.8 in P. gymnocercus and $77.8 \%$ in C. thous) and an Acantocephala of the genus Centrorhynchus was also observed in $5.6 \%$ of $C$. thous only. These results indicated the helminths fauna in wild canids from the studied area.
\end{abstract}

KEY WORDS: Wild canids, helminths fauna, nematodes, trematodes, cestodes.

\section{RESUMO}

Quarenta canídeos selvagens foram capturados por "live trap" nos municípios de Pedro Osório e Pelotas, sul do estado do Rio Grande do Sul e transportados para o Laboratório de Parasitologia da Universidade Federal de Pelotas. Após serem necropsiados, segmentos do intestino, respiratório, urinário e

\footnotetext{
${ }^{1}$ Laboratório Regional de Diagnóstico, Faculdade de Veterinária (FV), Universidade Federal de Pelotas (UFPel), Pelotas, RS 96010-900. Brasil. E-mail: ruas@ufpel.edu.br

${ }^{2}$ Departamento de Microbiologia e Parasitologia, UFPel, RS.

${ }^{3}$ Programa de Pós-Graduação em Veterinária. FV, UFPel, RS.

${ }^{4}$ Departamento de Clínica Médica Veterinária. Faculdade de Agronomia e Medicina Veterinária, Universidade Federal de Mato Grosso, Cuibá, MT.
}

fígado foram separados e examinados. Os crânios dos animais foram usados para identificação taxonômica. Dos 40 animais capturados, 22 (55\%) foram Pseudalopex gymnocercus e 18 (45\%) Cerdocyon thous. Os nematóides mais prevalentes foram: Ancylostoma caninum (45,4 em $P$. gymnocercus e $22,2 \%$ em C. thous), Molineus felineus (9,9 em $P$. gymnocercus e 5,6\% em C. thous), Strongyloides sp. (22,7 em P. gymnocercus e 16,7\% em C. thous), Trichuris sp. (13,6 em P. gymnocercus e 11,1\% em C. thous), e Capillaria hepatica (13,6 em P. gymnocercus e 5,5\% em C. thous). Os trematódeos observados foram: Alaria alata $(50,0 \% \mathrm{em} C$. thous e 36,4 em P. gymnocercus), e Asthemia heterolecithodes em 5,6\% dos C. thous. Cestóides foram identificados como Spirometra sp. (61,1\% em C. thous e 54,5 em P. gymnocercus), Diphyllobothriidae (81,8 em P. gymnocercus e 77,8\% em C. 
thous), e Acantocephala do gênero Centrorhynchus foi observado somente em 5,6\% dos C. thous. Estes resultados indicaram a helmintofauna de canídeos selvagens nas áreas estudadas.

PALAVRAS-CHAVE: canídeos selvagens, helmintofauna, nematóides, trematódeos, cestóides.

\section{INTRODUÇÃO}

Na América do Sul ocorrem sete gêneros e 11 espécies de canídeos silvestres. Destas, três habitam o Estado do Rio Grande do Sul, Pseudalopex gymnocercus (Fischer, 1814) (cachorro-do-campo), Cerdocyon thous (Linnaeus, 1766) (cachorrodo-mato) e Chrysocyon brachyurus Illiger, 1811 (lobo-guará) (BERTA, 1982; SILVA, 1994; MACDONALD, 1993; GONZÁLEZ, 2001). O lobo-guará, por estar na Lista Nacional das Espécies Ameaçadas de Extinção do Ministério do Meio Ambiente, não foi incluído nesse trabalho. Schmidt e Martin (1978), na Província do Chaco Boreal Paraguaio, relataram pela primeira vez Spirometra mansonoides (Mueller, 1935) parasitando C. thous e P. gymnocercus. Segundo Curtis (1980) o diagnóstico definitivo de Diphyllobothriidae só é possível por meio de infecções experimentais de plerocercóides em hospedeiros susceptíveis e com posterior recuperação do parasito adulto. Martinéz $(1986,1987)$ e Martinéz et al. (2000) no nordeste Argentino, identificaram Athesmia foxi Goldberger e Crane, 1911, Ancylostoma caninum (Ercolani, 1959) e Diphyllobothrium sp. (Cobbold, 1858), parasitando o intestino delgado de P. gymnocercus. O conhecimento dos parasitos que ocorrem nos animais silvestres de determinada região é de fundamental importância para o estabelecimento de programas de controle. A crescente utilização de áreas que servem de habitat para várias espécies silvestres, para fins de produção agropecuária e novos assentamentos urbanos, têm aumentado a possibilidade de contato entre animais silvestres e domésticos, favorecendo a transposição de parasitos de um hospedeiro a outro. O objetivo do presente trabalho foi investigar a prevalência de helmintos em canídeos silvestres do sul do Estado do Rio Grande do Sul.

\section{MATERIAL E MÉTODOS}

Foram capturados 40 canídeos silvestres nos municípios de Pedro Osório ( $32^{\circ} 01$ ' $28^{\prime \prime}$ "de latitude Sul, $52^{\circ} 55^{\prime} 01$ " de longitude W a $68 \mathrm{~m}$ de altitude) e Pelotas $\left(31^{\circ} 35^{\prime} 23,7^{\prime \prime}\right.$ ' de latitude Sul, $52^{\circ} 20^{\prime} 40,8^{\prime \prime}$ de longitude $\mathrm{W}$ a $36 \mathrm{~m}$ de altitude) com autorização do Instituto Brasileiro de Meio Ambiente e Recursos Naturais Renováveis - IBAMA através das licenças de coleta $n^{\circ}$. 112/99 e 022/2002, utilizando-se armadilhas modelo "Live Trap". Os animais foram eutanasiados com pentobarbital sódico por via endovenosa conforme Conselho Federal de Medicina Veterinária (2002). As necropsias foram realizadas segundo a técnica preconizada por Barros (1988). Fragmentos de fígado com alterações macroscópicas foram coletados, fixados em solução de formol a $10 \%$, posteriormente incluídos em parafina, cortados em micrótomo a $5 \mu \mathrm{m}$ e corados com hematoxilina-eosina. As porções do trato digestório foram abertas em bandeja, o conteúdo lavado em série de tamises malha $0,5 \mathrm{~mm}$ e $0,15 \mathrm{~mm}$ simultaneamente. $\mathrm{O}$ material retido nos tamises foi fixado em AFA e posteriormente inspecionado ao estereomicroscópio (até 40X). Os trematódeos, cestóides e acantocéfalos coletados foram mantidos resfriados entre 4 e $8^{\circ} \mathrm{C}$ por $24 \mathrm{~h}$ para distensão. Após, foram comprimidos entre lâminas e fixados em solução de AFA (Álcool 70 GL-93\%; Formol-5\%; Ácido Acético Glacial-2\%) frio por $48 \mathrm{~h}$. Após este período, foram retirados das lâminas e mantidos em AFA por mais 24h e depois conservados em álcool $70^{\circ} \mathrm{GL}$ glicerinado. O processamento dos trematódeos, cestóides e acantocéfalos foi realizado de acordo com a técnica descrita por Amato (1985). Os nematóides foram clarificados pelo lactofenol e montados temporariamente entre lâmina e lamínula para identificação, posteriormente foram classificados de acordo com as chaves de Yamaguti (1961), Anderson, Chabaud e Willmott (1974-1982), Anderson e Chabaud (1983) e Vicente et al. (1997). Os trematódeos foram classificados segundo Skrjabin (1964), Travassos et al. (1969), Yamaguti (1958; 1971) e Thatcher (1993). Os cestóides foram classificados conforme Yamaguti (1959) e Schmidt (1986). Os acantocéfalos foram classificados de acordo com Yamaguti (1963) e Petrochenko (1971). Os parâmetros analisados foram: prevalência, abundância, intensidade e intensidade média de parasitismo (MARGOLIS et al., 1982). As espécies de canídeos silvestres foram diferenciadas anatomicamente segundo Macdonald (1993) e Gonzáles (2001).

\section{RESULTADOS}

Dos 40 canídeos capturados, 22 (55\%) eram $P$. gymnocercus e 18 (45\%) eram C. thous. Quanto aos helmintos, constatou-se a presença de 14 espécies, sendo sete de nematóides, três de cestóides, dois acantocéfalos e dois trematódeos. Todos os animais estavam infectados por pelo menos uma espécie de helminto, sendo encontradas de uma a oito espécies infectando simultaneamente os hospedeiros, com média de 5,11 espécies por hospedeiro. Em P. gymnocercus observaram-se de uma a oito espécies de parasitos, com média de cinco por hospedeiro e em C. thous de duas a sete espécies, com média de 5,11 por hospedeiro. Não foi observada a presença de Echinococcus granulosus Batsch, 1796. A prevalência e a intensidade média de helmintos nos animais estudados são apresentadas nas Tabelas 1 e 2, respectivamente. Nos cortes histológicos dos fígados com alterações macroscópicas, observaram-se lesões multifocais caracterizadas por pontos amarelados distribuídos aleatoriamente na superfície capsular. Histologicamente, as lesões apresentavam poucos agregados multifocais de ovos com morfologia compatível aos de Capillaria. Ao redor desses ovos observou-se infiltrado inflamatório mononuclear, com raros eosinófilos e mínima proliferação de fibroblastos. Em alguns campos foram observados vários aglomerados de ovos, cortes transversais e longitudinais de parasitos adultos com presença de infiltrado inflamatório e alguns focos de calcificação. As alte- 
Tabela 1. Prevalência (\%) de helmintos em carnívoros silvestres Pseudolapex gymnocercus e Cerdocyon thous na região sul do Rio Grande do Sul, Brasil.

\begin{tabular}{|c|c|c|c|c|c|c|c|}
\hline \multirow[t]{2}{*}{ Helminto } & \multicolumn{4}{|c|}{ P. gymnocercus } & \multicolumn{3}{|c|}{ C. thous } \\
\hline & $\begin{array}{c}\mathrm{TC} \\
(\mathrm{n}=40)\end{array}$ & $\begin{array}{c}\mathrm{TPg} \\
(\mathrm{n}=22)\end{array}$ & $\begin{array}{c}\text { Machos } \\
(n=13)\end{array}$ & $\begin{array}{c}\text { Fêmeas } \\
(n=9)\end{array}$ & $\begin{array}{c}\text { TCt } \\
(n=18)\end{array}$ & $\begin{array}{c}\text { Machos } \\
(n=10)\end{array}$ & $\begin{array}{c}\text { Fêmeas } \\
(n=8)\end{array}$ \\
\hline \multicolumn{8}{|l|}{ Filo Nematoda } \\
\hline Molineus felineus & 7,50 & 9,09 & 15,38 & 0,00 & 5,56 & 10,00 & 0,00 \\
\hline Ancylostoma caninum & 35,00 & 45,45 & 38,46 & 55,56 & 22,22 & 30,00 & 12,50 \\
\hline Capillaria hepatica & 10,00 & 13,64 & 23,08 & 0,00 & 5,56 & 10,00 & 0,00 \\
\hline Capillaria sp. & 2,50 & 0,00 & 0,00 & 0,00 & 5,56 & 0,00 & 12,50 \\
\hline Trichuris sp. & 12,50 & 13,64 & 0,00 & 33,33 & 11,11 & 10,00 & 12,50 \\
\hline Strongyloides sp. & 20 & 22,73 & 15,38 & 33,33 & 16,67 & 20,00 & 12,50 \\
\hline Physalopteridae & 17,50 & 9,09 & 15,38 & 0,00 & 27,78 & 10,00 & 50,00 \\
\hline \multicolumn{8}{|l|}{ Classe Cestoda } \\
\hline Spirometra sp. & 57,50 & 54,55 & 53,85 & 55,56 & 61,11 & 70,00 & 50,00 \\
\hline Diphyllobothriidae & 80,00 & 81,82 & 92,31 & 66,67 & 77,78 & 90,00 & 62,50 \\
\hline Cyclophyllidea & 7,50 & 4,55 & 7,69 & 0,00 & 11,11 & 0,00 & 25,00 \\
\hline \multicolumn{8}{|l|}{ Classe Trematoda } \\
\hline Athesmia heterolecithodes & 2,50 & 0,00 & 0,00 & 0,00 & 5,56 & 12,50 & 0,00 \\
\hline Alaria alata & 42,50 & 36,36 & 46,15 & 22,22 & 50,00 & 50,00 & 50,00 \\
\hline \multicolumn{8}{|l|}{ Filo Acanthocephala } \\
\hline Centrorhynchus sp. & 2,50 & 0,00 & 0,00 & 0,00 & 5,56 & 0,00 & 12,50 \\
\hline Acanthocephala & 5,00 & 4,55 & 7,69 & 0,00 & 5,56 & 0,00 & 12,50 \\
\hline
\end{tabular}

TC=total capturados;

$\mathrm{TPg}=$ total de $P$. gymnocercus capturados;

$\mathrm{TCt}=$ total de $C$. thous capturados.

Tabela 2. Intensidade Média de helmintos em carnívoros silvestres Pseudolapex gymnocercus e Cerdocyon thous na região sul do Rio grande do Sul, Brasil.

\begin{tabular}{|c|c|c|c|c|c|c|c|}
\hline \multirow[t]{2}{*}{ Helminto } & \multicolumn{4}{|c|}{ P. gymnocercus } & \multicolumn{3}{|c|}{ C. thous } \\
\hline & $\begin{array}{c}\mathrm{TC} \\
(\mathrm{n}=40)\end{array}$ & $\begin{array}{c}\mathrm{TPg} \\
(\mathrm{n}=22)\end{array}$ & $\begin{array}{c}\text { Machos } \\
(n=13)\end{array}$ & $\begin{array}{c}\text { Fêmeas } \\
(n=9)\end{array}$ & $\begin{array}{c}\mathrm{TCt} \\
(\mathrm{n}=18)\end{array}$ & $\begin{array}{c}\text { Machos } \\
(\mathrm{n}=10)\end{array}$ & $\begin{array}{c}\text { Fêmeas } \\
(n=8)\end{array}$ \\
\hline \multicolumn{8}{|l|}{ Filo Nematoda } \\
\hline Molineus felineus & 2,00 & 1,50 & 1,50 & 0,00 & 3,00 & 3,00 & 0,00 \\
\hline Ancylostoma caninum & 1,36 & 1,30 & 1,40 & 1,20 & 1,50 & 1,67 & 1,00 \\
\hline Capillaria hepatica & 1,00 & 1,00 & 1,00 & 0,00 & 1,00 & 1,00 & 0,00 \\
\hline Capillaria sp. & 1,00 & 0,00 & 0,00 & 0,00 & 1,00 & 0,00 & 1,00 \\
\hline Trichuris sp. & 1,80 & 2,00 & 0,00 & 2,00 & 1,50 & 1,00 & 2,00 \\
\hline Strongyloides sp. & 1,63 & 1,40 & 1,50 & 1,33 & 2,00 & 2,50 & 1,00 \\
\hline Physalopteridae & 1,43 & 1,50 & 1,50 & 0,00 & 1,40 & 1,00 & 1,50 \\
\hline \multicolumn{8}{|l|}{ Classe Cestoda } \\
\hline Spirometra sp. & 2,87 & 2,67 & 3,14 & 2,00 & 3,09 & 3,00 & 3,25 \\
\hline Diphyllobothriidae & 5,63 & 5,00 & 5,67 & 3,67 & 6,43 & 6,44 & 6,40 \\
\hline Cyclophyllidea & 1,33 & 2,00 & 2,00 & 0,00 & 1,00 & 0,00 & 1,00 \\
\hline \multicolumn{8}{|l|}{ Classe Trematoda } \\
\hline Athesmia heterolecithodes & 1,00 & 0,00 & 0,00 & 0,00 & 1,00 & 0,00 & 1,00 \\
\hline Alaria alata & 2,18 & 2,00 & 1,83 & 2,50 & 2,33 & 2,40 & 2,25 \\
\hline \multicolumn{8}{|l|}{ Filo Acanthocephala } \\
\hline Centrorhynchus sp. & 2,00 & 0,00 & 0,00 & 0,00 & 2,00 & 0,00 & 2,00 \\
\hline Acanthocephala & 2,00 & 2,00 & 2,00 & 0,00 & 2,00 & 0,00 & 2,00 \\
\hline
\end{tabular}

TC=total capturados;

$\mathrm{TPg}=$ total de $P$. gymnocercus capturados;

$\mathrm{TCt}=$ total de $C$. thous capturados.

rações histológicas observadas, hospedeiro, órgão de eleição e morfologia dos ovos sugerem que $10 \%$ dos canídeos estavam parasitados por C. hepatica (Bancroft, 1893). Os valores para $P$. gymnocercus e $C$. thous variaram em estimativa de prevalência, $13,64 \%$ e 5,56 \%, respectivamente. A intensidade média para este parasito não pode ser avaliada, visto que a deteç̧ão desta espécie foi feita através da visualização do parasito em cortes histológicos do fígado. No trato respiratório dos canídeos não foi observada a presença de parasitos.

No estômago foram encontrados parasitos da Ordem Spirurida, Família Physalopteridae, enquanto que no intestino delgado constatou-se a presença de Strongyloides sp. 
Grassi, 1879, Ancylostoma caninum, Spirometra sp. Faust, Campbell e Kellogg, 1929, Alaria alata Goeze, 1782, além de espécimes de Cyclophyllidea, Diphyllobothriidae. No intestino grosso constatou-se a presença de Trichuris sp. Roederer, 1761 Strongyloides sp. e exemplares da Família Diphyllobothriidae. Na vesícula biliar encontrou-se Athesmia heterolecithodes (Braun, 1899) e na bexiga um exemplar de Capillaria sp.

\section{DISCUSSÃO}

De acordo com estudos realizados no Brasil e países da América Latina (MARTINÉZ et al., 2000; RUAS et al., 2003; VIEIRA et al., 2004), canídeos silvestres $P$. gymnocercus e $C$. thous são hospedeiros de um importante número de nematóides, cestóides e trematódeos. A prevalência de parasitos intestinais pode variar devido a fatores tais como: região geográfica, comportamento do hospedeiro, estação do ano e composição da população dos hospedeiros (LABARTHE et al., 2004). Só foi possível o diagnóstico em nível de Família e Ordem dos cestóides devido ao estádio de desenvolvimento dos espécimes, que apresentavam escólex e o estróbilo com poucas proglótides em início de desenvolvimento, não permitindo a visualização de órgãos genitais, os quais são fundamentais no enquadramento taxonômico dos exemplares. Vários autores têm observado estes cestóides parasitando canídeos sul-americanos, além de outros carnívoros como Felis geoffroyi, F. yagouaroundi e Procyon cancrivorus (SCHMIDT; MARTIN, 1978; MARTINÉZ et al., 2000; RUAS et al., 2002). A origem da infecção se deve, possivelmente, à dieta dos hospedeiros definitivos em seu ambiente natural, sendo freqüente o consumo de peixes, crustáceos de água doce, répteis e pequenos mamíferos, que atuariam como hospedeiros intermediários ou paratênicos dos parasitos (SILVA, 1994; DOTTO, 2001; JÁCOMO et al., 2004). Na Argentina, há citação de Diphyllobothrium em Lontra provocax, onde foi citado também parasitando várias espécies de peixes de água doce (REVENGA et al., 1995; SATO et al., 1999), que, segundo Bueno e Mota-Júnior (2004) também fazem parte da dieta de P. gymnocercus e C. thous. Parasitismo por A. alata foi observado em $42,5 \%$ dos animais. Estes valores são semelhantes aos encontrados por Shimalov e Shimalov (2002) em Vulpes vulpes $(42,6 \%)$ na Bielorrussia e são superiores aos valores encontrados por Rigonatto et al. (2004) na Venezuela, que observaram uma prevalência de $16,6 \%$ e $12,5 \%$, respectivamente em $P$. gymnocercus e $C$. thous. Este trematódeo é comumente encontrado no intestino delgado de carnívoros como canídeos, felídeos, mustelídeos e procionídeos. Necessita de ambiente com presença de áreas com boa irrigação, que favoreça o desenvolvimento de seus hospedeiros intermediários, na sua maioria compostos de anfíbios e moluscos (RIGONATTO et al., 2004), e componentes da dieta dos canídeos silvestres estudados (DOTTO, 2001). Segovia et al. (2002) relataram $19,2 \%$ de A. alata em V. vulpes na Península Ibérica, considerando que a área de ocorrência dos hospedeiros era possivelmente um foco natural de trematodíases zoonóticas. Considerando-se que suínos selvagens podem se infectar e albergar metacercárias, atuariam como hospedeiros intermediários ou paratênicos de A. alata (SLAVICA et al., 2002) e servindo de fonte de infecção tanto para os canídeos silvestres como para o homem. Situação semelhante pode ocorrer na região em estudo, onde o javali (Sus scrofa ferus) encontra-se disseminado, podendo atuar como fonte de infecção. Parasitismo por $A$. heterolecithodes foi encontrado somente em C. thous e com baixa prevalência geral, 2,5\%. Este trematódeo apresenta baixa especificidade pelos hospedeiros, já tendo sido encontrado parasitando uma ampla gama de grupos zoológicos (PAULSEN et al, 1999; DIGIANI, 2000). O diagnóstico do parasitismo por A. caninum e Strongyloides sp. pode ser explicado pela presença do hospedeiro preferencial Canis familiaris na área de captura dos animais. Nesta região é cultural e funcional, o uso de cães no manejo de campo com os rebanhos ovino e bovino, o que determina que estes animais se desloquem em habitat dos canídeos silvestres, havendo uma sobreposição de nichos entre espécies de canídeos silvestres e domésticos (DOTTO, 2001) e com isso a possibilidade de infecção cruzada entre os hospedeiros, assim como pelas próprias fezes, que tem sua dispersão maximizada pela característica comportamental de demarcação de território com fezes e urina (COOPER, 2003). Existem, relativamente, poucos trabalhos sobre a helmintofauna de canídeos silvestres na Região Neotropical e nenhum autor até o momento relatou a presença do gênero Molineus Cameron, 1923. Segundo Durette-Desset e Chabaud (1981) o gênero Molineus parasitaria carnívoros de várias partes do mundo e também primatas não humanos neotropicais. A prevalência de $C$. hepatica $(10 \%)$ foi observada em canídeos adultos, possivelmente, causada pelo aumento da exposição a ovos embrionados e também devido ao regime alimentar destes animais, que inclui pequenos roedores e seus cadáveres (CROWELL et al. 1978). O parasitismo por Centrorhynchus sp. (Luhe, 1911) apresentou prevalência geral de 2,5\%. É um acantocéfalo com grande número de espécies (PETROCHENKO, 1971) e está presente em outro hospedeiro da região em estudo, Didelphis albiventris com prevalência de $40 \%$ (MÜLLER, 2005). A ocorrência de parasitos identificados em nível de Filo Acanthocephala e do gênero Centrorhynchus em Canideos silvestres é justificada pela dieta dos hospedeiros que, apesar de serem nominados como carnívoros, apresentam uma dieta onívora, incluindo uma grande quantidade de vertebrados e invertebrados que podem atuar como hospedeiros intermediários ou paratênicos de acantocephalos (GOLDBERG; BURSEY, 2003; DOTTO, 2001; JÁCOMO, 2004).

Uma grande diversidade de helmintos foi encontrado nas duas espécies de canídeos estudadas. Muitos desses parasitos são reconhecidos por estarem presentes nas populações de cães domésticos, e alguns deles possuem potencial direto ou indireto de risco a saúde pública. Os nematóides são os helmintos predominantes em $P$. gymnocercus e C. thous, com $100 \%$ dos animais parasitados e, C. hepatica, M. felineus e A. 
alata são espécies registradas pela primeira vez no Brasil em P. gymnocercus e C. thous.

\section{REFERÊNCIAS BIBLIOGRÁFICAS}

AMATO, J. F. R.. Manual de Técnicas para a Preparação de Coleções Zoológicas. 8. Platelmintos (Temnocefálidos, Trematódeos, Cestóides, Cestodários) e Acantocéfalos, Sociedade Brasileira de Zoologia, São Paulo, 1985. 11 p.

ANDERSON, R.C.; CHABAUD, A.G. CIH Keys to the nematode parasites of vertebrates, Farnham Royal, England: CAB, n. 10, 1983, 86 p.

ANDERSON, R.C.; CHABAUD, A.G.; WILLMOTT, S. CIH Keys to the nematode parasites of vertebrates. Farnham Royal: CAB, n. 1-9, 1974-1982, 467 p.

BARROS, C.S.L. Guia de necropsia dos mamíferos domésticos. Santa Maria: Biblioteca Central da UFSM, 1988. 48 p.

BERTA, A. Cerdocyon thous. Mammalian Species, v. 186, p. 1-4, 1982.

BUENO, A.A.; MOTTA-JÚNIOR, J.C. Food habits of two syntopic canids, the maned wolf (Chrysocyon brachyuros) and the crab-eating fox (Cerdocyon thous), in southeastern Brazil. Revista Chilena Historia Natural, v. 77, n. 1, p. 514, 2004.

CONSELHO FEDERAL DE MEDICINA VETERINÁRIA DO BRASIL. 2002. Resolução CFMV n ${ }^{0} 714$, de 20 de junho de 2002.

COOPER, T. 2003. "Pseudalopex gymnocercus" (On-line), Animal Diversity Web. Disponível em: <http:// animaldiversity.ummz.umich.edu/site/accounts/ information/Pseudalopex_gymnocercus.html $>$. Acesso em: 22 jan. 2005.

CROWELL, W.A.; KLEI, T.R.; HALL, D.I. Capillaria hepatica infection in coyotes of Louisiana. Journal American Veterinary Medical Association, v. 173, n. 9, p. 1171-1172, 1978.

CURTIS, M.A. Problems in reporting Diphyllobothrium latum (fish tapeworms) in Canada on the basis of de stool sample analysis. Canadian Diseases Vector Reporter, v. 6, p. 4950, 1980.

DIGIANI, M.C. Digeneans and cestodes parasitic in the whitefaced ibis Plegadis chihi (Aves: Threskiornithidae) from Argentina. Folia Parasitologica, v. 47, n. 3, p. 195-204, 2000.

DOTTO, J.C.; FABIAN, M.E.; MENEGHETI, J.O. Atuação de Pseudalopex gymnocercus (Fischer, 1814) e de Cerdocyon thous (Linnaeus, 1776) (Mammalia Canidae) como fator de mortalidade de cordeiros no sul do Brasil. Boletin de la Sociedad de Biologia de Concepcion, v. 72, p. 44 - 52, 2001.

DURETTE-DESSET, M.C.; CHABAUD, A.G. Sur les Molineinae parasites de Mammiferes. Annales de Parasitologie Humaine et Comparée, v. 56, n. 3, p. $297-$ 312, 1981.
GOLDBERG, S.R.; BURSEY, C.R. Helminths of two anuran species, Atelopus spurrelli (Bufonidae) and Dendrobates histrionicus (Dendrobatidae), from Colombia, South America. Parasitology International, v.52, n.3 p. 251-253, 2003.

GONZÁLEZ, E.M. Guía de Campo de los Mamíferos de Uruguay. Introducción al Estudio de los Mamíferos, Montevideo: Vida Silvestre, 2001. 339p.

JÁCOMO, A.T. de A.; SILVEIRA, L.; DINIZ FILHO, J.A.F. Niche separation between the maned wolf (Chrysocyon brachyurus), the crab-eating fox (Dusycion thous) and the hoary fox (Dusicyon vetulus) in central Brazil. Journal of Zoology (London), v. 262, n. 1, p. 99-106, 2004.

LABARTHE, N.; SERRÃO, M.L.; FERREIRA, A.M.R.; ALMEIDA, N.K.O.; GUERRERO, J. A survey of gastrointestinal helminths in cats of the metropolitan region of Rio de Janeiro, Brazil. Veterinary Parasitology, v.133,n. 2-3, p. 133-139, 2004.

MACDONALD, D. The Encyclopedia of Mammals, New York: Fact on File, 1993. p. 517-524.

MARGOLIS, L.; ESCH, G.W.; HOLMES, J.C.; KURIS, A.M.; SCHAD, G.A. The use of ecological terms in parasitology (report of an ad doc committee of the American Society of Parasitologistis). Journal of Parasitology, v. 68, n. 1, 131133, 1982.

MARTINÉZ, F.A. Helmintofauna de los mamiferos silvestres. Trematodes. Veterinaria Argentina, v. 3, n. 26, p. 544548, 1986.

MARTINÉZ, F.A. Zooparasitos de mamiferos silvestres. Veterinaria Argentina, v. 4, n. 33, p. 266-271, 1987.

MARTINÉZ, F.A.; TROIANO, J.C.; GAUNA. A.L.; DUCHENE, A.; JUEGA-SICARDI, J.A. Frecuencia de infección por Diphyllobothrium sp. (Cestoda: Diphyllobothriidae) en carnívoros silvestres de Argentina. Boletin Chileno de Parasitología, v. 55, n. 3-4., p. 100103, 2000.

MÜLLER, G. Diversidade e potencial zoonótico de parasitos de Didelphis albiventris Lund, 1841. 2005. 139f. Tese (Doutorado em Ciências Veterinárias)- Faculdade de Veterinária/UFRGS, Porto Alegre, 2005.

PAULSEN, R.M.M.; MULLER, G.; BRUM, J.G.W.; SINKOC, A.L. Hydrochoeristrema massaroi n. sp. (trematoda: paramphistomidae: clanorchiimi) parasito de ratão-do-banhado (myocastor coypus). Arquivos do Instituto Biológico, São Paulo, v. 66, n. 1, p. 135-137, 1999.

PETROCHENKO, V.J. Acanthocephala of domestic and wild animals.. Jerusalém. Keter Press, 1971. v. 1 e 2. 952p.

REVENGA, J.E.; PERFUMO, C.; UBEDA, C.A.; SEMENAS, L. Difilobotriasis en salmónidos introducidos en el Parque y Reserva Nacional Nahuel Huapi, Argentina: Patologia de las lesiones provocadas por Diphyllobothrium spp. Archivos de Medicina Veterinaria, v. 23, n.2 p. 115121, 1995.

RIGONATTO, T.; MARTINÉZ, F.A.; NUNEZ, S.E.; STANCATO, M.R.; TROIANO, J.C.; GAUNA, A.L.; 
DUCHENE, A.; STANCATO, M.R.; JUEGA-SICARDI, J.A. 2004. Hallazgo de Alaria sp. (Trematoda, Strigeiidae) en carnívoros silvestres. Corrientes, Argentina. Disponível em: <http://www.unne.edu.ar/cyt/2000/4_veterinarias/ v_pdf $/$ v_040.pdf $>$. Acesso em: 12 dez. 2004.

RUAS, J.L.; MULLER, G.; CASTRO, E.J.; BRUM, J.G.W.; BERNE, M.E.A.; FARIAS, N.A.R. Primeiro relato de Diphyllobothrium sp. e Spirometra sp. em Lycalopex gymnocercus cachorro-do-campo) no Rio Grande do Sul. In: CONGRESSO DA SOCIEDADE DE ZOOLÓGICOS DO BRASIL, 26, 2002, Porto Alegre. Anais... Porto Alegre: SZB, 2002. p. 177.

RUAS, J.L.; SOARES, M.P.; FARIAS, N.A.R.; BRUM, J.G.W. Infecção por Capillaria hepatica em Carnívoros Silvestres (Lycalopex gymnocercus e Cerdocyon thous) na Região Sul do Rio Grande do Sul. Arquivos do Instituto Biológico de São Paulo, v.70, n. 2, p. 147-150, 2003.

SATO, H.; IHAMA, Y.; INABA, T.; YAGISAWA, M.; KAMIYA, H. Helminth fauna of carnivores distributed in North-Western Tohoku, Japan, with special reference to Mesocestoides paucitesticulos and Brachylaima tokudai. Journal of Veterinary Medicine, v. 61, n. 12, p. 1339-1342, 1999.

SCHMIDT, G.D. CRC Handbook of Tapeworm Identification, Miami: CRC Press, 1986, 675p.

SCHMIDT, G.; MARTIN, R.L. Tapewormes of the Chaco Boreal, Paraguay, with two new species. Journal of Helmintology, v. 52, n. 3, p. 205-209, 1978.

SEGOVIA, J.M.; TORRES, J.; MIQUEL, J. The red fox, Vulpes vulpes L., as a potential reservoir of zoonotic flukes in the Iberian Península. Acta Parasitologica, v. 47, n. 2, p. 163-166, 2002.

SHIMALOV, V.V. : SHIMALOV, V.T. Helminth fauna of the red fox (Vulpes vulpes Linnaeus, 1758) in southern Belarus. Parasitology Research, v. 89, n. 1, p. 77-78, 2002.
SILVA, F. Mamíferos Silvestres do Rio Grande do Sul. Porto Alegre: FZB Rio Grande do Sul, 1994. 246p.

SKRJABIN, K.I. Keys to the Trematodes of Animals and Man. Urbana: University of Illinois Press, 1964. 351p.

SLAVICA, J.; UHITIL, S.; MARIJA, V. Mesocercariae of fluke Alaria alata determined in wild boar meat. Zeitschrift für Jagdwissenschaft, v. 48, n.3, p. 203-207, 2002.

THATCHER, V.E. Trematódeos Neotropicais.Manaus: INPA, 1993. 553p.

TRAVASSOS, L.; FREITAS, J.F.T.; KOHN, A. Trematódeos do Brasil. Memórias do Instituto Oswaldo Cruz, tomo 67, p. 1-886, 1969. Fascículo único.

VIEIRA, F.M.; HORTA-DUARTE, F.; LOUZADA, G.L.; BESSA, E.C.A.; SOUZA LIMA, S. Aspectos da ecologia de helmintos em Cerdocyon thous Linnaeus, 1766 (Carnívora: Canidae) na Zona da Mata Mineira. In: CONGRESSO BRASILEIRO DE ZOOLOGIA, 25, 2004, Brasília. Resumos. Brasília: Sociedade Brasileira de Zoologia, 2004. p.462.

VICENTE, J. J.; RODRIGUES, H. O.; GOMES, D. C.; PINTO, R. M. Nematóides do Brasil. Parte V: Nematóides de mamíferos. Revista Brasileira de Zoologia. v. 14, (supl.1), p. 1-452, 1997.

YAMAGUTI, S. Systema Helminthum. The Digenetic Trematodes of Vertebrates. New York: Interscience Publishers, Inc., 1958. v..I,1575 p.

YAMAGUTI, S. Systema Helminthum. The Cestodes of Vertebrates, New York: Interscience Pub. Inc., 1959. v. III $880 \mathrm{p}$.

YAMAGUTI, S. Systema Helminthum. The Nematodes of Vertebrates. New York: Interscience Pub. Inc., 1961. v. III, $679 \mathrm{p}$.

YAMAGUTI, S. Systema Helmintum: Acanthocephala. New York:Interscience, 1963. $423 \mathrm{p}$.

YAMAGUTI, S. Synopsis of Digenetic Trematodes of Vertebrates. Tokyo: Keigaku, 1971. 2 v. 1074p.

Recebido em 21 de maio de 2007.

Aceito para publicação em 30 de junho de 2008 . 